\title{
Characteristics of unplanned hospitalisations among cancer patients in Singapore
}

Qingyuan Zhuang ${ }^{1}{ }_{M M E D}(F M)$, Joanna SE $\underline{\text { Chan }}{ }^{2}{ }_{M M E D}(E M)$, Lionel KY See ${ }^{3}{ }^{3}{ }^{M E D}(I M)$, Jianbang $\underline{\text { Chiang }}{ }^{4}{ }_{M M E D}(I M)$, Shariff R $\underline{\text { Suhaimi }}{ }^{5}{ }_{M R C P}$, Tallie WL $\underline{\text { Chua }}{ }^{2}{ }_{M M E D ~(E M)}$, Anantharaman Venkataraman ${ }^{2}{ }_{\text {FRCSEd (A\&E) }}$

\begin{abstract}
Introduction: Cancer is a pervasive global problem with significant healthcare utilisation and cost. Emergency departments (EDs) see large numbers of patients with oncologic emergencies and act as "gate-keepers" to subsequent hospital admissions. A proportion of such hospital admissions are rapidly discharged within 2 days and may be potentially avoidable.

Methods: Over a 6-month period, we conducted a retrospective audit of active cancer patients presenting to the ED with subsequent admission to the Department of Medical Oncology. Our aims were to identify independent factors associated with a length of stay $\leq 2$ days; and characterise the clinical and resource needs of these short admissions.

Results: Among all medical oncology admissions, 24.4\% were discharged within 2 days. Compared to longer stayers, patients with short admissions were significantly younger $(P=0.010)$, had lower National Early Warning Scores (NEWS) $(P=0.006)$, and had a lower proportion of gastrointestinal and hepatobiliary cancers $(P=0.005)$. Among short admissions, common presenting medical problems were infections $(n=144,36.3 \%)$, pain $(n=116,29.2 \%)$, gastrointestinal complaints $(n=85,21.4 \%)$ and respiratory complaints $(\mathrm{n}=76,19.1 \%)$. These admissions required investigations and treatments already available at the ED.

Conclusion: Short admissions have low resource needs and may be managed in the ED. This may help save valuable inpatient bed-days and reduce overall healthcare costs.
\end{abstract}

Ann Acad Med Singap 2021;50:882-91

Keywords: Emergency medicine, healthcare use, oncology, palliative care, unplanned cancer admission

\section{INTRODUCTION}

Cancer is a pervasive global problem with growing healthcare utilisation and costs. ${ }^{1-3}$ This situation is similar in Singapore where cancer incidence is on the rise and accounts for nearly $30 \%$ of total population mortality. ${ }^{4,5}$ Singapore data suggests that cancer patients accounted for $13 \%$ of total healthcare costs in 2016, with a major portion driven by hospitalisations. ${ }^{6}$ Notably, cancer care remains hospital-centric at the end of life (EOL) with high healthcare cost per capita, recurrent emergency department (ED) visits, recurrent hospitalisations and many deaths in acute-care hospitals. ${ }^{6-9}$
Hospital-based EDs assess and manage cancer patients with a wide range of oncologic emergencies. They are typically the "gate-keepers" to subsequent hospital admissions. Knowledge regarding cancer-related utilisation of emergency care and the subsequent clinical course is vital to identify gaps in care and opportunities for service development to improve health outcomes. ${ }^{10}$ Several international studies have described characteristics of cancer-related acute healthcare use, with infections and poorly controlled symptoms being key drivers of ED visits and subsequent hospital admissions. ${ }^{11-14}$ In Singapore, limited

\footnotetext{
${ }^{1}$ Division of Supportive and Palliative Care, National Cancer Centre, Singapore

${ }^{2}$ Division of Emergency Medicine, Singapore General Hospital, Singapore

${ }^{3}$ Department of Internal Medicine, Singapore General Hospital, Singapore

${ }^{4}$ Division of Medical Oncology, National Cancer Centre, Singapore

${ }^{5}$ Singhealth Community Hospital, Singapore

Correspondence: Dr Qingyuan Zhuang, Division of Supportive and Palliative Care, National Cancer Centre Singapore, 11 Hospital Crescent, Singapore 169610.

Email: zhuang.qingyuan@singhealth.com.sg
} 


\section{CLINICAL IMPACT}

\section{What is New}

- Nearly a quarter of cancer admissions to the Division of Medical Oncology at Singapore General Hospital are relatively stable, have low resource needs, and may be potentially managed without inpatient admission.

\section{Clinical Implications}

- These findings can potentially guide ongoing efforts to develop clinical protocols for greater ambulatory management of selected cancer patients to reduce hospitalisations and save valuable inpatient bed-days.

information is currently available on the characteristics of cancer patients presenting to the ED, their care and subsequent outcomes. ${ }^{7,15}$ To fill this gap, we embarked on a long-term undertaking to study the management of oncology-related emergencies at the ED (MORE-ED) in a retrospective cohort of cancer patients. ${ }^{16}$

One of the goals of MORE-ED was to identify oncologic presentations where inpatient admissions could be potentially avoidable. Studies done in other communities have alluded to the need to identify avoidable cancer admissions. ${ }^{17-20}$ However, generalisability of findings is difficult with a lack of definition of "avoidable", as these may be influenced by complex contextual factors such as availability of community support, social and cultural pressures and prompt access to specialist ambulatory care. ${ }^{21-23}$ Following observations from a recent prospective multicentre cohort study that 25\% of admissions have a length of stay (LOS) $\leq 2$ days and may potentially be avoided, ${ }^{12}$ we set out to examine our centre's oncologic presentations with LOS of no more than 2 days. Once an ED patient becomes an inpatient, many hospital resources are committed, including clerking, reviews by ward teams, more investigations and usually twice daily reviews and decision-making resulting in most such patients staying for $>1$ day. ${ }^{24}$ These admissions may be potentially avoided if alternative models of care are available. For example, multiple and frequent reviews for up to 6 or 8 times within a 23-hour time frame can be instituted, as have been done in many hospital emergency observation units for non-cancer and cancer populations. ${ }^{24-28}$

Patients presenting to the ED and admitted as inpatients with a LOS of $\leq 2$ days are usually less complex and potentially dischargeable via streamlined ambulatory management pathways. ${ }^{29}$ Thus, our aims were to identify independent factors associated with a LOS $\leq 2$ days; and characterise the clinical and resource needs of these short admissions from the ED to the Division of Medical Oncology (DMO).

\section{METHODS}

\section{Setting, study design and participants}

Our study was set within SingHealth Outram Campus, which houses the 1,723-bed Singapore General Hospital (SGH) and the National Cancer Centre Singapore (NCCS). NCCS manages up to $70 \%$ of Singapore's public sector cancer patients. SGH has a dedicated adult ED with an annual oncology-related attendance of about 12,000 patient visits, of whom more than $90 \%$ become inpatients. ${ }^{16}$

The MORE-ED study was conducted as an audit of ED management of oncology patients. The study relied on hospital electronic medical records and used both automated extraction of structured Electronic Health Records data via the Electronic Health Intelligence System, ${ }^{30}$ a platform built specifically for data management, and manual extraction of free-text by study team members.

Patients included in the study were active cancer patients who visited SGH ED and then subsequently admitted to DMO from 1 October 2018 to 31 March 2019. The patients were identified by extracting inpatient diagnosis-related group codes corresponding to one of the International Classification of Diseases (ICD) 10 codes for malignant neoplastic disease for all ED admissions to DMO. We excluded admissions with ED presentations that were not related to cancer, as well as admissions by patients with no active cancer or with carcinoma in situ. Active cancer was defined as cancer not provided potentially curative treatment; or with evidence that treatment has not been curative (e.g. recurrent or progressive disease); or with ongoing treatment. ${ }^{31}$ From these cancer admissions, we identified those who had a total hospital LOS of no more than 2 days for more in-depth data extraction and analysis.

\section{Study procedures and measures}

The data collection form was developed jointly by the ED, DMO and the Division of Supportive and Palliative Care study team members. Our planned unit of analysis was by patient visit instead of unique cancer patients, as 1 unique patient can have multiple visits for different reasons over the study period.

Patient variables captured included demographics, cancer type, prior cancer therapy, current oncological treatment intent, EOL discussions, readmissions within 
30 days and mortality records. For oncological treatment intent, patients on systemic antineoplastic treatment intended to achieve cure were categorised as "curative intent". Patients on systemic antineoplastic treatment intended to prolong life but not achieve cure were categorised as "palliative intent". Cancer patients no longer receiving systemic antineoplastic treatment were categorised as "best supportive care". Constituents of best supportive care included a broad range of treatments such as palliative care, symptom management and physical therapy. ${ }^{32}$
ED-related variables captured included triage categories, National Early Warning Score (NEWS), problem lists, investigations done, and treatments provided. NEWS is a scoring tool that aids prediction of clinical deterioration in adult patients ${ }^{33}$ (Table 1). Admission-related variables captured included diagnosis codes, investigations done, treatment given and disposition.

Treatments were categorised by pharmacologic class (fluids, non-opioid analgesics, opioid analgesics, antibiotics and procedures), investigations by blood

Table 1. National Early Warning Score (NEWS)

\begin{tabular}{|c|c|c|}
\hline Variable & Value & Score \\
\hline \multirow[t]{5}{*}{ Respiratory rate (breaths per minute) } & $\leq 8$ & +3 \\
\hline & $9-11$ & +1 \\
\hline & $12-20$ & 0 \\
\hline & $21-24$ & +2 \\
\hline & $\geq 25$ & +3 \\
\hline \multirow[t]{4}{*}{ Oxygen saturations (\%) } & $\leq 91$ & +3 \\
\hline & $92-93$ & +2 \\
\hline & $94-95$ & +1 \\
\hline & $\geq 96$ & 0 \\
\hline \multirow[t]{2}{*}{ Use of supplemental oxygen } & No & 0 \\
\hline & Yes & +2 \\
\hline \multirow[t]{5}{*}{ Temperature $\left({ }^{\circ} \mathrm{C}\right)$} & $\leq 35$ & +3 \\
\hline & $35.1-36$ & +1 \\
\hline & $36.1-38$ & 0 \\
\hline & $38.1-39$ & +1 \\
\hline & $\geq 39.1$ & +2 \\
\hline \multirow[t]{5}{*}{ Systolic blood pressure (mmHg) } & $\leq 90$ & +3 \\
\hline & $91-100$ & +2 \\
\hline & $101-110$ & +1 \\
\hline & $111-219$ & 0 \\
\hline & $\geq 220$ & +3 \\
\hline \multirow[t]{6}{*}{ Heart rate (beats per minute) } & $\leq 40$ & +3 \\
\hline & $41-50$ & +1 \\
\hline & $51-90$ & 0 \\
\hline & $91-110$ & +1 \\
\hline & $111-130$ & +2 \\
\hline & $\geq 131$ & +3 \\
\hline \multirow[t]{2}{*}{ Level of consciousness } & Alert & 0 \\
\hline & Responds to voice, responds to pain or unresponsive & +3 \\
\hline
\end{tabular}


tests (standard haematological and biochemical, blood cultures, and non-standard tests), imaging (e.g. plain $\mathrm{X}$-ray, computed tomography [CT] imaging, ultrasound, etc.) and other non-blood tests (e.g. urine and stool cultures, etc.). Standard blood tests included a full blood count, the renal panel and/or a troponin $\mathrm{T}$ test. All other haematological and biochemical blood investigations were classified as non-standard.

\section{Statistical analysis}

We compared descriptive statistics between ED visits with resulting short and long admissions. Differences in means were compared using one-way ANOVA. Differences in medians were compared using KruskalWallis test. Differences in proportions were compared using chi-square test or Fisher's Exact test as appropriate. Multivariable logistic regression was conducted using ED visit variables to identify associations with a subsequent short admission outcome. Because of the retrospective nature of this study, the NEWS variable had $25.2 \%$ missing data. The impact of missing data on the association with short admissions was evaluated via sensitivity analysis in which the NEWS variable was dropped from the regression model. No imputation was performed.

Stata/SE version 16.1 (StataCorp, College Station, US) was used to perform statistical analysis. A 2-sided $P<0.05$ was considered statistically significant.

\section{Research ethics and patient consent}

This study was approved by the SingHealth Institutional Review Board (CIRB Reference Number 2019/2554) as an audit of health services.

\section{RESULTS}

A total of 1,624 cancer patients were admitted to DMO by SGH ED from 1 October 2018 to 31 March 2019. Of these, 99 stayed for $<1$ day and 298 for $1-2$ days. Table 2 summarises the distribution of each independent variable by LOS.

\section{Patient characteristics}

Among all DMO admissions, patients with a LOS of $\leq 2$ days were younger $(P=0.010)$. Ethnic distribution in this sample closely reflected general population make-up. ${ }^{34}$

The majority $(63.0 \%)$ of DMO-admitted patients visited the ED after office hours (weekends and weekdays $5 \mathrm{pm}$ to $8 \mathrm{am}$ ). There was no significant difference in ED after-hours consultation between those who stayed $\leq 2$ days and those who stayed longer.
Generally, these patients spent $119 \pm 67.3$ minutes in the ED.

The 3 most common primary cancer sites were lung, gastrointestinal and breast, closely reflecting the most common cancers in Singapore. A higher proportion of patients with gastrointestinal and hepatobiliary cancers experienced longer inpatient stays, while a higher proportion of those with lung cancers stayed $\leq 2$ days $(P=0.005)$.

\section{Patient acuity at the ED}

There were no significant differences in the distribution of patients among the 3 triage categories between the 2 groups of patients $(P=0.402)$. NEWS scores were significantly higher among patients with LOS of $>2$ days $(P=0.006)$.

After multivariable logistic regression analysis, older age (odds ratio [OR] 0.99 per year increase, 95\% confidence interval [CI] 0.97-0.99), high NEWS (OR $0.35,95 \%$ CI $0.21-0.60$ ), and gastrointestinal and hepatobiliary cancers (OR $0.56,95 \%$ CI 0.38 0.83 ) remained significantly less likely to discharge within 2 days.

\section{Characteristics of short oncology admissions}

Among all patients admitted from the ED to the DMO, $397(24.4 \%)$ had a LOS of $\leq 2$ days (Table 3 ). Of these short oncology admissions, patients with metastatic cancer constituted $76 \%(n=302)$. Only $83 \quad(20.9 \%)$ visits were by patients still on curative treatment. Another 222 (55.9\%) were on palliative treatment and $92(23.3 \%)$ on best supportive care.

\section{Management at the ED for those with short hospital stay}

The common problems identified for admission were infections $(\mathrm{n}=144,36.3 \%)$, gastrointestinal symptoms $(\mathrm{n}=85,21.4 \%)$, pain $(\mathrm{n}=116,29.2 \%)$ and respiratory difficulty $(\mathrm{n}=76,19.1 \%)$ (Table 4). Examples of infections included pneumonia and hepatobiliary sepsis. Gastrointestinal problems included constipation, intestinal obstruction and ascites. Respiratory difficulties included pleural effusion, haemoptysis and breathlessness.

Almost all ED visits resulted in a standard haematological and biochemical panel $(\mathrm{n}=366,92.2 \%)$. Two hundred and seventy-two patients $(68.5 \%)$ received a plain $\mathrm{X}$-ray and $130(32.8 \%)$ underwent blood culture investigations. Common treatments provided included intravenous fluids $(\mathrm{n}=184,46.4 \%)$, antibiotics $(\mathrm{n}=126$, $31.7 \%)$, analgesia $(n=79,19.9 \%)$ and other symptomatic medications $(\mathrm{n}=130,32.8 \%)$. CT brain was performed 


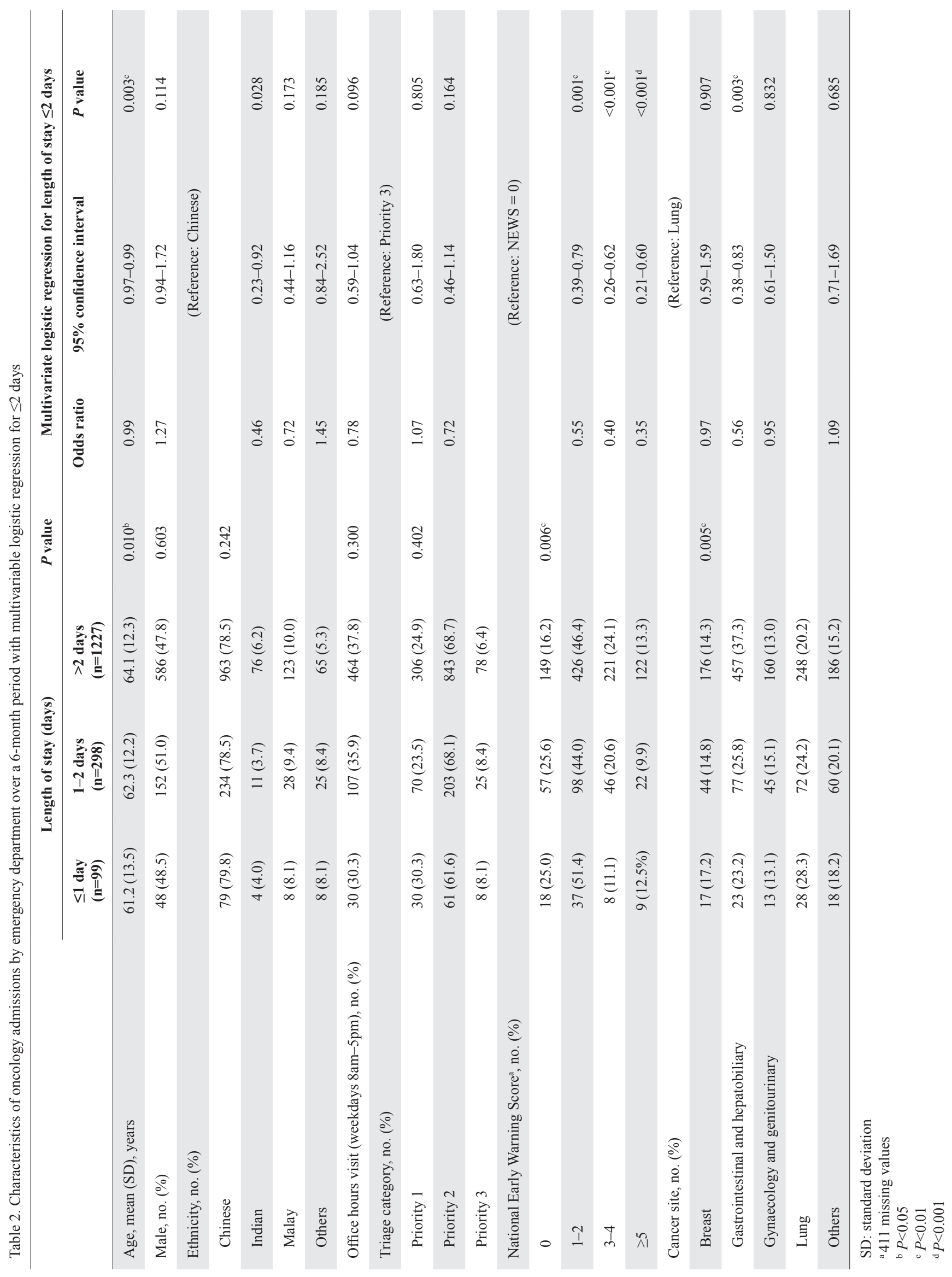


Table 3. Cancer characteristics among admissions with length of stay $\leq 2$ days

\begin{tabular}{|c|c|}
\hline Variables & $\begin{array}{c}N=397 \\
\text { No. }(\%)\end{array}$ \\
\hline \multicolumn{2}{|l|}{ Current treatment intent } \\
\hline Curative intent & $83(20.9)$ \\
\hline Palliative intent & $222(55.9)$ \\
\hline Best supportive care & $92(23.2)$ \\
\hline \multicolumn{2}{|l|}{ Prior cancer treatment received } \\
\hline Radiotherapy & $157(39.6)$ \\
\hline Chemotherapy & $281(70.8)$ \\
\hline Immunotherapy & $52(13.1)$ \\
\hline Surgery & $163(41.1)$ \\
\hline Targeted therapy/Hormones & $169(42.6)$ \\
\hline \multicolumn{2}{|l|}{ Cancer site } \\
\hline Breast & $61(15.4)$ \\
\hline Gastrointestinal & $63(15.9)$ \\
\hline Hepatobiliary & $37(9.3)$ \\
\hline Gynaecological and genitourinary & $58(14.6)$ \\
\hline Head and neck & $29(7.3)$ \\
\hline Lung & $100(25.2)$ \\
\hline Lymphoma & $22(5.5)$ \\
\hline Others & $27(6.8)$ \\
\hline Metastatic disease & $302(76)$ \\
\hline Prior documented EOL discussion & $100(25.2)$ \\
\hline EOL discussion conducted in ED & $24(6.1)$ \\
\hline
\end{tabular}

ED: emergency department; EOL: end-of-life

for $29(7.3 \%)$ patients and other CT imaging for $3(0.8 \%)$. Magnetic resonance imaging was not available in our ED.

Of the 116 patients with an identified pain problem at the ED, $37(31.9 \%)$ received neither opioid nor nonopioid analgesia. Of these, 19 subsequently received analgesia after admission. Of the 144 patients with an identified sepsis problem, $18(12.5 \%)$ did not receive any antibiotics while at the ED. Of these, 17 went on to receive antibiotics during the inpatient stay. Of the 43 patients who died in hospital or were terminally discharged, only $18(41.9 \%)$ had a documented EOL discussion in the ED. The remaining 25 patients received EOL discussion after admission.
Table 4. Emergency department problem list and resource use among admissions with length of stay $\leq 2$ days

\begin{tabular}{lc}
\hline Variables & $\begin{array}{c}\mathbf{N}=\mathbf{3 9 7} \\
\mathbf{N o}(\mathbf{\%})\end{array}$ \\
\hline Problem list & $144(36.3)$ \\
\hline Infection/Sepsis & $116(29.2)$ \\
\hline Pain & $32(8.1)$ \\
\hline Cardiac & $85(21.4)$ \\
\hline Gastrointestinal & $76(19.1)$ \\
\hline Respiratory & $9(2.3)$ \\
\hline Vascular & $16(4.0)$ \\
\hline Genitourinary & $43(10.8)$ \\
\hline Neurological & $11(2.8)$ \\
\hline Renal and electrolytes & $1(0.3)$ \\
\hline Bone & $6(1.5)$ \\
\hline Side effects of cancer treatment & $0(0)$ \\
\hline Post-surgical complications & $7(1.8)$ \\
\hline Device issues & $47(11.8)$ \\
\hline Others & \\
\hline Pen & \\
\hline
\end{tabular}

Investigations

\begin{tabular}{|c|c|}
\hline Standard haematological tests ${ }^{\mathrm{a}}$ & $366(92.2)$ \\
\hline Standard biochemical tests ${ }^{\mathrm{b}}$ & $368(92.7)$ \\
\hline Blood cultures & $130(32.8)$ \\
\hline X-ray & $272(68.5)$ \\
\hline CT brain & $29(7.3)$ \\
\hline Other CT imaging & $3(0.8)$ \\
\hline Non-standard blood investigations & $61(15.4)$ \\
\hline \multicolumn{2}{|l|}{ Treatments } \\
\hline Intravenous fluids & $184(46.4)$ \\
\hline Non-opioid analgesia & $22(5.5)$ \\
\hline Opioid analgesia & $79(19.9)$ \\
\hline Antibiotics & $126(31.7)$ \\
\hline Other symptomatic medications & $130(32.8)$ \\
\hline Vasopressors & $3(0.8)$ \\
\hline Intubation & $2(0.5)$ \\
\hline Other procedures & $4(1.0)$ \\
\hline Onsite palliative review & $0(0)$ \\
\hline
\end{tabular}

CT: computed tomography

${ }^{\text {a }}$ Full blood count

${ }^{\mathrm{b}}$ Renal panel, liver panel, cardiac enzymes 


\section{Further management after admission}

Upon admission, 71 (17.9\%) received additional nonstandard haematological and/or biochemical tests (Table 5). About one-fifth $(\mathrm{n}=81,20.4 \%)$ received additional bacteriological investigations such as urine cultures. Only a small proportion of admissions required specialised radiological imaging such as CT and MRI. Another 10 patients were identified as having sepsis. Treatments instituted included intravenous fluids in $170(42.8 \%)$ and antibiotics in $154(38.8 \%)$. EOL discussion was documented in $85(21.4 \%)$ patients during the short admission. Palliative care consult was provided to 32 patients $(8.1 \%)$, while 349 patients (87.9\%) were discharged home with follow-up arrangements. Among those discharged alive, 101 (28.1\%) were readmitted within 30 days.

\section{DISCUSSION}

The ED sees many active cancer patients with a high proportion being subsequently admitted. Similar to recent findings by Caterino et al., almost a quarter of these admissions were successfully discharged within 2 days at the SGH. ${ }^{12}$ These patients may possibly have been managed more aggressively within the ED or other similar short-stay facility, with use of purposefully designed structured clinical protocols, careful patient selection and good clinical reasoning, while maintaining quality and safety of care..$^{27-29}$ This preliminary study aimed to define the study population that could benefit. The majority (63\%) of such ED visits by cancer patients took place after working hours. This suggests that such care protocols should be available and accessible even after office hours and on weekends. Clinical observations protocols, such as those available in most EDs for a variety of non-cancer emergencies, may be suitable approaches for managing such patients. Our findings also showed that low NEW scores at ED triage (especially a score of 0 ) were significantly associated with a shorter hospitalisation. A recent Singapore study on use of NEWS in an acute medical ward attested to its ability to accurately triage patients according to the likelihood of adverse outcomes in infection-related acute medical settings..$^{33}$ Low NEW scores could be one potential trigger for placing patients on observation protocols in the ED.

Common presenting symptoms for short oncology admissions included pain, breathlessness, gastrointestinal symptoms and fever. This is similar to findings from other countries. ${ }^{12-14}$ Management plans for all these symptoms can be initiated and instituted at EDs, especially for haemodynamically stable patients, and can potentially be relieved within several hours. ED observations units would have the resources to manage such complaints. ${ }^{25}$ Most patients admitted to DMO required investigations easily accessible at the ED. Observation protocols could potentially include repeating some of these investigations to document normalisation of test results, if so required. Even for the $20 \%$ of patients in our study cohort who required nonstandard blood tests such as CT, ultrasound, inflammatory markers and bacteriological investigations such as urine cultures, these could, with appropriate arrangements, be performed in short-stay observation units.

Most short oncology admissions required intravenous fluids, analgesics, other symptomatic medications and antibiotics. However, a proportion did not receive prompt symptomatic treatment at the ED setting and instead received treatment only after admission. Implementation of treatment protocols at the ED may reduce delays in symptomatic care, and if coupled with intensive short-term observation and frequent reviews within a 24-hour period, could possibly help reduce avoidable hospitalisations. ${ }^{35,36}$

EDs are usually busy environments and ED physicians may not be able to devote time to conducting long consultations requiring EOL discussions and palliative care arrangements. Dedicated and trained nurse case managers may be able to fulfil these roles and help manage selected oncology patients without hospital admission. Oncology departments can also initiate such discussions with their patients in the early stages of disease management, rather than only after they have been admitted from the ED for a cancer-related problem. Oncology and palliative physicians can work closely with emergency physicians in facilitating these discussions within ED short-stay units. A multicentre clinical trial is currently being conducted across the US that aims to develop and support primary palliative care within the ED through multicomponent interventions. ${ }^{37}$ Adapting some of these approaches may help address the currently low $6.1 \%$ EOL discussion rate at the ED and $25.1 \%$ rate of prior EOL discussions by oncology. These discussions should also be systematically recorded and easily accessible to all downstream providers to facilitate revisiting when needed. ${ }^{37}$ Useful materials from resources such as VitalTalk and Serious Illness Conversation Guide can be adapted as needed..$^{38,39}$

Readmissions of cancer patients after initial discharge is not unexpected. Metastatic cancer readmission rates of $16.5 \%$ had been previously reported by Low et al. ${ }^{40}$ 
Table 5. Inpatient resource use, disposition, and readmissions among admissions with length of stay $\leq 2$ days

\begin{tabular}{|c|c|}
\hline Variables & $\begin{array}{c}\mathrm{N}=397 \\
\text { No. }(\%)\end{array}$ \\
\hline \multicolumn{2}{|l|}{ Investigations } \\
\hline Standard haematological tests ${ }^{\mathrm{a}}$ & $133(33.5)$ \\
\hline Standard biochemical tests ${ }^{\mathrm{b}}$ & $280(70.5)$ \\
\hline Blood cultures & $36(9.1)$ \\
\hline Plain X-ray & $47(11.8)$ \\
\hline CT brain & $10(2.5)$ \\
\hline Other CT imaging & $30(7.6)$ \\
\hline Nonstandard haematological or biochemical tests & $71(17.9)$ \\
\hline Magnetic resonance imaging & $6(1.5)$ \\
\hline PET scan & $0(0)$ \\
\hline Ultrasound scan & $5(1.3)$ \\
\hline Cancer markers & $16(4.0)$ \\
\hline Other cultures (other than blood) & $81(20.4)$ \\
\hline \multicolumn{2}{|l|}{ Treatments } \\
\hline Intravenous fluids & $170(42.8)$ \\
\hline Non opioid analgesics & $88(22.2)$ \\
\hline Opioid analgesics & $117(29.5)$ \\
\hline Antibiotics & $154(38.8)$ \\
\hline Other symptomatic medications & $191(48.1)$ \\
\hline Interventional radiology and endoscopy & $8(2.0)$ \\
\hline Inpatient palliative review & $32(8.1)$ \\
\hline Surgery & $1(0.3)$ \\
\hline Inpatient chemotherapy & $5(1.3)$ \\
\hline Inpatient radiotherapy & $0(0)$ \\
\hline EOL discussion documented during admission & $85(21.4)$ \\
\hline \multicolumn{2}{|l|}{ Disposition } \\
\hline Death in hospital & $38(9.6)$ \\
\hline Discharged with follow-up & $349(87.9)$ \\
\hline Terminal discharge & $5(1.3)$ \\
\hline Transferred to inpatient hospice & $2(0.5)$ \\
\hline Discharge against medical advice/Abscond & $3(0.8)$ \\
\hline Readmission within 30 days (excluding death in hospital, total $n=359$ ) & $101(28.1)$ \\
\hline
\end{tabular}

CT: computed tomography; EOL: end-of-life; PET: positron emission tomography

${ }^{a}$ Full blood count

${ }^{\mathrm{b}}$ Renal panel, liver panel, cardiac enzymes 
There will be a need to study characteristics of the $28.1 \%$ of patients in our group who were readmitted within 30 days. Short hospital LOS cannot be at the expense of higher readmission rates as fragmented and repeated care transitions due to premature discharges are detrimental to good patient management. Processes should be embedded to identify patients at higher risk for 30-day readmissions so that proactive multicomponent interventions such as home palliative service, case management, medication review and caregiver support ${ }^{41}$ may be implemented early.

\section{Limitations}

Our study had some limitations inherent to retrospective studies. Our sample included only ED visits by cancer patients from a single tertiary public sector hospital in Singapore. However, our hospital is affiliated to the NCCS, which sees $70 \%$ of all public sector cancer patients in Singapore. Our findings may be considered representative of public sector cancer care and relevant for other similar institutions in the region.

We had a sizable proportion of incomplete NEWS scores $(25.3 \%)$, which were excluded from analysis and presumed to be missing at random. This missing information was equally distributed with $25.2 \%$ missing NEWS in long admissions and $25.7 \%$ missing NEWS in short admissions. Excluding NEWS from the sensitivity analysis, the OR for the rest of the variables remained similar as those for the full model. (Table S1 of Supplementary Appendix in the online version of this article.)

We were unable to capture cancer characteristics, ED management and inpatient management data for the longer hospitalisations. We were also unable to capture important sociodemographic characteristics such as caregiver stress, socioeconomic status, social support, key cancer characteristics such as performance status, metastatic burden, current cancer treatment, comorbidity severity and laboratory results. Some of these factors may be associated with longer hospitalisations. More research is needed to develop risk stratification algorithms that could accurately identify cancer patient groups more amenable to rapid management, observation and discharge without admission.

\section{CONCLUSION}

A quarter of all unplanned oncology hospitalisations by SGH ED are rapidly discharged within 2 days. Patients who are younger, have low NEWS score, and have non-gastrointestinal and hepatobiliary cancers are more likely to experience a short admission.
Short admissions have lower resource needs and may potentially be managed in an ambulatory setting. Consideration is needed for the development of protocols for more aggressive initial care and frequent reviews of selected cancer patients within the ED. This may help save valuable inpatient bed-days and reduce overall healthcare costs.

\section{REFERENCES}

1. Prager GW, Braga S, Bystricky B, et al. Global cancer control: responding to the growing burden, rising costs and inequalities in access. ESMO Open 2018;3:e000285.

2. Park J, Look KA. Health Care Expenditure Burden of Cancer Care in the United States. Inquiry 2019;56:46958019880696.

3. Bekelman JE, Halpern SD, Blankart CR, et al. Comparison of Site of Death, Health Care Utilization, and Hospital Expenditures for Patients Dying With Cancer in 7 Developed Countries. JAMA 2016;315:272-83.

4. Data.gov.sg. Number of Deaths and Top 10 Principal Causes. Available at: https://data.gov.sg/dataset/principal-causes-ofdeath?resource_id\%3D98d62914-67a3-47c8-bd7a-310fb3b07a0f. Accessed on 14 September 2021.

5. Cancer Registry - National Registry Of Diseases Office. Available at: https://www.nrdo.gov.sg/publications/cancer. Accessed on 14 September 2021.

6. Yan S, Kwan YH, Thumboo J, et al. Characteristics and Health Care Utilization of Different Segments of a Multiethnic Asian Population in Singapore. JAMA Netw Open 2019;2:e1910878.

7. Wong TH, Lau ZY, Ong WS, et al. Cancer patients as frequent attenders in emergency departments: A national cohort study. Cancer Med 2018;7:4434-6.

8. Tan WS, Bajpai R, Low CK, et al. Individual, clinical and system factors associated with the place of death: A linked national database study. PLoS ONE 2019;14:e215566.

9. Zhuang Q, Lau ZY, Ong WS, et al. Sociodemographic and clinical factors for non-hospital deaths among cancer patients: A nationwide population-based cohort study. PLoS ONE 2020;15:e0232219.

10. Brown J, Grudzen C, Kyriacou DN, et al. The Emergency Care of Patients With Cancer: Setting the Research Agenda. Ann Emerg Med 2016;68:706-11.

11. Rivera DR, Gallicchio L, Brown J, et al. Trends in Adult CancerRelated Emergency Department Utilization. JAMA Oncol 2017;3:e172450.

12. Caterino JM, Adler D, Durham DD, et al. Analysis of Diagnoses, Symptoms, Medications, and Admissions Among Patients With Cancer Presenting to Emergency Departments. JAMA Netw Open 2019;2:e190979.

13. Gallaway MS, Idaikkadar N, Tai E, et al. Emergency department visits among people with cancer: Frequency, symptoms, and characteristics. J Am Coll Emerg Physicians Open 2021;2:e12438.

14. Hong AS, Sadeghi N, Harvey V, et al. Characteristics of Emergency Department Visits and Select Predictors of Hospitalization for Adults With Newly Diagnosed Cancer in a Safety-Net Health System. J Oncol Pract 2019;15:e490-500.

15. Tan AL, Iyer NG, Putri N, et al. Factors driving frequent attendance at emergency departments for patients with head and neck cancer. Head \& Neck 2019;41:3798-805. 
16. Tallie Chua WL, Chan JSE, Lai G, et al. Management of Oncology-Related Emergencies at the Emergency Department (MORE-ED): A Long-Term Undertaking. J Emerg Med 2019; $57: 423$.

17. Brooks GA, Abrams TA, Meyerhardt JA, et al. Identification of potentially avoidable hospitalizations in patients with GI cancer. J Clin Oncol 2014;32:496-503.

18. Brooks GA, Jacobson JO, Schrag D. Clinician Perspectives on Potentially Avoidable Hospitalizations in Patients With Cancer. JAMA Oncol 2015;1:109-10.

19. Numico G, Cristofano A, Mozzicafreddo A, et al. Hospital Admission of Cancer Patients: Avoidable Practice or Necessary Care? PLoS ONE 2015;10:e0120827.

20. Johnson PC, Xiao Y, Wong RL, et al. Potentially Avoidable Hospital Readmissions in Patients With Advanced Cancer. J Oncol Pract 2019;15:e420-7.

21. Chen H, Walabyeki J, Johnson M, et al. An integrated understanding of the complex drivers of emergency presentations and admissions in cancer patients: Qualitative modelling of secondary-care health professionals' experiences and views. PLoS ONE 2019; 14:e0216430.

22. O'Cathain A, Knowles E, Turner J, et al. Variation in avoidable emergency admissions: multiple case studies of emergency and urgent care systems. J Health Serv Res Policy 2016;21:5-14.

23. Lynch B, Fitzgerald AP, Corcoran P, et al. Drivers of potentially avoidable emergency admissions in Ireland: an ecological analysis. BMJ Qual Saf 2019;28:438-48.

24. Wright B, Martin GP, Ahmed A, et al. How the Availability of Observation Status Affects Emergency Physician Decisionmaking. Ann Emerg Med 2018;72:401-9.

25. Lateef F, Anantharaman V. The short-stay emergency observation ward is here to stay. Am J Emerg Med 2000;18:629-34.

26. Gatto A, Rivetti S, Capossela L, et al. Utility of a pediatric observation unit for the management of children admitted to the emergency department. Ital J Pediatr 2021;47:11.

27. Lipitz-Snyderman A, Klotz A, Atoria CL, et al. Impact of Observation Status on Hospital Use for Patients With Cancer. J Oncol Pract 2015;11:73-7.

28. Brooks GA, Chen EJ, Murakami MA, et al. An ED pilot intervention to facilitate outpatient acute care for cancer patients. Am J Emerg Med 2016;34:1934-8
29. Handley NR, Schuchter LM, Bekelman JE. Best Practices for Reducing Unplanned Acute Care for Patients With Cancer. J Oncol Pract 2018;14:306-13.

30. Electronic Health Intelligence System. Available at: https://www.ihis. com.sg/Project_Showcase/Healthcare_Systems/Pages/eHINTS.aspx. Accessed on 12 July 2021.

31. Kearon C, Ageno W, Cannegieter SC, et al. Categorization of patients as having provoked or unprovoked venous thromboembolism: guidance from the SSC of ISTH. J Thromb Haemost 2016;14:1480-3.

32. Lester JF, Agulnik J, Akerborg O, et al. What constitutes best supportive care in the treatment of advanced non-small cell lung cancer patients?--Results from the lung cancer economics and outcomes research (LUCEOR) study. Lung Cancer 2013;82:128-35.

33. Lim WT, Fang AH, Loo CM, et al. Use of the National Early Warning Score (NEWS) to Identify Acutely Deteriorating Patients with Sepsis in Acute Medical Ward. Ann Acad Med Singap 2019;48:145-9.

34. gov.sg. What are the racial proportions among Singapore citizens? 10 December 2019. Available at: https:/www.gov.sg/article/whatare-the-racial-proportions-among-singapore-citizens. Accessed on 7 December 2021 .

35. Silva FF, da Silva Bonfante GM, Reis IA, et al. Hospitalizations and length of stay of cancer patients: A cohort study in the Brazilian Public Health System. PLoS ONE 2020;15:e0233293.

36. Perry M, Franks N, Pitts SR, et al. The impact of emergency department observation units on a health system. Am J Emerg Med 2021;48:231-7.

37. Grudzen CR, Brody AA, Chung FR, et al. Primary Palliative Care for Emergency Medicine (PRIM-ER): Protocol for a Pragmatic, Cluster-Randomised, Stepped Wedge Design to Test the Effectiveness of Primary Palliative Care Education, Training and Technical Support for Emergency Medicine. BMJ Open 2019;9:e030099.

38. VitalTalk. Available at: https://www.vitaltalk.org/. Accessed on 10 May 2021.

39. Ariadne Labs. Serious Illness Conversation Guide. Available at: https://www.ariadnelabs.org/areas-of-work/serious-illness-care/ resources/. Accessed on 10 May 2021.

40. Low LL, Liu N, Wang S, et al. Predicting 30-Day Readmissions in an Asian Population: Building a Predictive Model by Incorporating Markers of Hospitalization Severity. PLoS ONE 2016;11:e0167413.

41. Kripalani S, Theobald CN, Anctil B, et al. Reducing Hospital Readmission: Current Strategies and Future Directions. Annu Rev Med 2014;65:471-85. 